\title{
Decomposition of Eshelby's energy momentum tensor and application to path and domain independent integrals for the crack extension force of a plane circular crack in Mode III loading
}

\author{
K. Eriksson
}

Received: 1 June 2006 / Accepted: 20 June 2007 / Published online: 24 July 2007

(C) Springer Science+Business Media B.V. 2007

\begin{abstract}
Vanishing divergence of Eshelby's (energy momentum) tensor allows formulation of path or domain independent integral expressions of the crack extension force. In this work, a decomposition scheme of this tensor is presented, which results in zero divergence decomposed parts that allow formulation of expressions yielding the Mode I, II and III crack tip parameters $J$ and $K$, with particular emphasis on Mode III, at present. By using the Mode III decomposed part of Eshelby's tensor and the virtual crack extension method, a path and a domain independent integral, both new, for the crack extension force of a plane circular crack in axi-symmetric Mode III loading, are derived as examples of application.
\end{abstract}

Keywords Path domain independent · Mode III . Axi-symmetric · Circular crack

\section{Introduction}

Two common ways to determine the in-plane crack extension force for a three-dimensional crack is to calculate the $J$-integral, either point-wise on a plane perpendicular to the crack front or as a mean over a

K. Eriksson $(\varangle)$

Department of Solid Mechanics,

Luleå University of Technology,

97187 Luleå,

Sweden

e-mail: kjell.eriksson@1tu.se domain enclosing some part of the crack front. The $J$-integral is usually expressed as a contour integral or a sum of a contour and an area integral in the former case and as a volume or domain integral in the latter. The various forms of the $J$-integral are very attractive in practice, because of path or domain independence of the pertaining integrals and relative ease of calculation with numerical methods. On the whole, contour and area integrals are most conveniently treated with the boundary element method (Rigby and Aliabadi 1993) and domain integrals, since long, with the finite element method (e.g. Li et al. 1985).

Irwin (1957) separated the crack tip near-field quantities into three different modes, Mode I-III, where Mode I is the crack opening mode, Mode II is the inplane shearing mode and Mode III is the out-of-plane shearing, or tearing mode. Mode I is symmetric and Mode II and III are anti-symmetric with respect to the plane of the crack.

In linear elasticity it is possible to determine stress intensity factors through calculation of the $J$-integral. For each mode there is a corresponding $J$-integral, $J^{\mathrm{I}}, J^{\mathrm{II}}$ and $J^{\mathrm{III}}$, which are related to $J$ as follows (Nikishkov and Atluri 1987)

$$
\begin{gathered}
J=J^{\mathrm{I}}+J^{\mathrm{II}}+J^{\mathrm{III}}=\frac{K_{\mathrm{I}}^{2}+K_{\mathrm{II}}^{2}}{E^{*}}+\frac{K_{\mathrm{III}}^{2}}{2 G} \\
\text { where } E^{*}=\left\{\begin{array}{ll}
E /\left(1-v^{2}\right) & \text { plane strain } \\
E & \text { plane stress }
\end{array}\right. \text { and } \\
\qquad G=\frac{E}{2(1+v)}
\end{gathered}
$$


and $E, v$ the elastic modulus and Poisson's ratio, respectively.

Basically, there are two different methods (ibid.) to obtain the stress intensity factors $K_{\mathrm{I}}, K_{\mathrm{II}}$ and $K_{\mathrm{III}}$ from $J$. The most general and numerically stable method (Huber et al. 1993), which is exploited here, is the decomposition method (Ishikawa et al. 1979). In this method, $J$ is first split into two parts $J=J^{S}+J^{A}$ where $J^{S}$ and $J^{A}$ are calculated from the symmetric and anti-symmetric elastic fields about the crack plane, respectively. The part $J^{S}$ is equal to the symmetric Mode I integral $J^{\mathrm{I}}$. The remaining part $J^{A}$ is then decomposed into the $J^{\mathrm{II}}$ - and $J^{\mathrm{III}}$-integrals (Nikishkov and Atluri 1987; Rigby and Aliabadi 1998), and finally the stress intensity factors can be obtained from $J^{\mathrm{I}}, J^{\mathrm{II}}$ and $J^{\mathrm{III}}$.

The $J$-integral may appear in several different forms and the most common are contour, area or domain integrals or combinations thereof, but other forms exist. Forms that exploit the energy momentum tensor (Eshelby 1951, 1970) are path or domain independent if the condition of a vanishing divergence of the momentum tensor is fulfilled. Then instead of first formulating various forms of the $J$-integral and then perform decomposition for each type, it is more convenient to once and for all determine the decomposition scheme of the momentum tensor into zero divergence partials. Path or domain independence is then inherent in integrals obtained through suitable integration and transformation operations on such partials.

The momentum tensor decomposition route is explored in this work, and with emphasis on Mode III loading. By using the Mode III decomposition of Eshelby's tensor and the virtual crack extension method (Eriksson 2000) a path and a domain independent integral for the crack extension force of a circular and axi-symmetrically loaded Mode III crack are derived.

The analysis yields results which agree with previously known solutions of the crack extension force in the particular case of an axi-symmetrically loaded circular crack. For a curved crack front, the $J$-integral expressions for a straight crack are supplemented by additive integral expressions in the form of an area integral and a volume integral for a point-wise and a domain-wise $J$, respectively. The effect of a crack front with constant radius of curvature upon such supplementary integrals is found to be inversely proportional to the radius of curvature.

\section{Decomposition of the energy momentum tensor}

The vanishing divergence of Eshelby's energy momentum tensor $P_{l j}$ allows formulation of path or domain independent integrals. For

$P_{l j}=W \delta_{l j}-\sigma_{j i} u_{i}$,

where $W$ is strain energy and $\delta_{i j}$ the Kronecker symbol, $\sigma_{i j}$ stress, $u_{i}$ displacement and a comma (,) denotes differentiation, we have for a strain energy function that depends only on strain, but not explicitly on coordinates, or $W=W\left(\varepsilon_{i j}\right)$, where $\varepsilon_{i j}$ is strain, that

$P_{l j},{ }_{j}=0$

that is, the divergence of Eshelby's tensor vanishes.

In the absence of a crack, the integral of (4) over some regular domain $V$

$\int_{V} P_{l j}, j \mathrm{~d} V=0$

that is, the integral vanishes independently of the domain $V$, as the integrand is zero everywhere. In other words, this integral, and transformations thereof, are path or domain independent. This property allows construction of other forms of path or domain independent integrals by using transformations of (5). The most common manipulations involve integration by parts and the divergence theorem. To prove path or domain independence of such transformations explicitly is often more cumbersome (e.g. Huber et al. 1993; Rigby and Aliabadi 1998) than the convenient direct use of (5).

To prove (4), we have, in short, for the terms in $P_{l j}$ the steps

$$
\left(W \delta_{l j}\right)_{, j}=\delta_{l j} W,{ }_{j}=W, l=\frac{\partial W}{\partial \varepsilon_{i j}} \varepsilon_{i j}, l=\sigma_{i j} u_{i},{ }_{j l}
$$

where we have used the property of the strain energy function $\sigma_{i j}=\partial W / \partial \varepsilon_{i j}$, the strain-displacement relationship $\varepsilon_{i j}=\left(u_{i},{ }_{j}+u_{j},{ }_{i}\right)$ and symmetry of stress $\sigma_{j i}=\sigma_{i j}$, on the one hand and

$$
\left(\sigma_{j i} u_{i}, l\right),{ }_{j}=\sigma_{i j},{ }_{j} u_{i}, l+\sigma_{j i} u_{i}, l j
$$

on the other.

For (6) and (7) to be equal the stress must be symmetric and satisfy equilibrium

$\sigma_{i j},{ }_{j}=0$ 
and further, the condition of commuting second derivatives of displacement

$u_{i}, j_{k}=u_{i}, k j$

must be satisfied. If all conditions are fulfilled, then (4) is satisfied and the divergence of Eshelby's tensor vanishes at every point.

Consider an integral over the domain $V$ with the integrand taken as (6) minus (7)

$$
\int_{V}\left(\sigma_{i j} u_{i},{ }_{j l}-\sigma_{i j},{ }_{j} u_{i}, l-\sigma_{j i} u_{i},{ }_{l j}\right) \mathrm{d} V
$$

then this integral is domain independent. This form is useful in that all conditions critical for domain independence in relation to decomposition are explicit. These conditions are equilibrium of stress and commutative displacement derivatives, the condition of stress symmetry always being fulfilled, as this condition is not affected in the decomposition process.

Consider a three-dimensional body with a plane crack whose front is straight or smoothly curved. A local coordinate system $\left(x_{1}, x_{2}, x_{3}\right)$ with origin at the tip of the crack is oriented such that $x_{2}$ is normal to the plane of the crack, $x_{3}$ tangent to the crack front and $x_{1}$ directed into the body.

It is well established (Ishikawa et al. 1979) that an entire field of stress, strain or displacement can be decomposed into a sum of mirror-type symmetric and anti-symmetric parts with respect to the local $x_{1}$ axis. For the stress and displacement fields we have

$$
\begin{aligned}
\sigma_{i j}= & \sigma_{i j}^{S}+\sigma_{i j}^{A} \\
= & \frac{1}{2}\left[\begin{array}{ccc}
\sigma_{11}+\sigma_{11}^{\prime} & \sigma_{12}-\sigma_{12}^{\prime} & \sigma_{13}+\sigma_{13}^{\prime} \\
\ldots & \sigma_{22}+\sigma_{22}^{\prime} & \sigma_{23}-\sigma_{23}^{\prime} \\
\text { sym. } & \ldots & \sigma_{33}+\sigma_{33}^{\prime}
\end{array}\right] \\
& +\frac{1}{2}\left[\begin{array}{ccc}
\sigma_{11}-\sigma_{11}^{\prime} & \sigma_{12}+\sigma_{12}^{\prime} & \sigma_{13}-\sigma_{13}^{\prime} \\
\ldots & \sigma_{22}-\sigma_{22}^{\prime} & \sigma_{23}+\sigma_{23}^{\prime} \\
\text { sym. } & \ldots & \sigma_{33}-\sigma_{33}^{\prime}
\end{array}\right]
\end{aligned}
$$

and

$$
u_{i}=u_{i}^{S}+u_{i}^{A}=\frac{1}{2}\left[\begin{array}{l}
u_{1}+u_{1}^{\prime} \\
u_{2}-u_{2}^{\prime} \\
u_{3}+u_{3}^{\prime}
\end{array}\right]+\frac{1}{2}\left[\begin{array}{l}
u_{1}-u_{1}^{\prime} \\
u_{2}+u_{2}^{\prime} \\
u_{3}-u_{3}^{\prime}
\end{array}\right]
$$

Here superscripts $S$ and $A$ denote the symmetric and anti-symmetric parts, respectively, of the stress and displacement fields. Un-primed symbols $\left(\sigma_{i j}\right.$, etc, ) denote a field quantity at a point $P=\left(x_{1}, x_{2}, x_{3}\right)$ while primed symbols $\left(\sigma_{i j}^{\prime}\right.$, etc. ) denote the quantity of the same field at the symmetric point $P^{\prime}=\left(x_{1},-x_{2}, x_{3}\right)$.
The stress fields $\sigma_{i j}$ and $\sigma_{i j}^{\prime}$ are related and in particular

$\sigma_{i j}^{\prime}\left(x_{1}, x_{2}\right)=\sigma_{i j}\left(x_{1},-x_{2}\right)$

$\sigma_{i j, 2}^{\prime}\left(x_{1}, x_{2}\right)=-\sigma_{i j, 2}\left(x_{1},-x_{2}\right)$

From (11) and the relations (13a, b), it was shown (ibid.) that

$\sigma_{i j}^{S},{ }_{j}=0$ and $\sigma_{i j}^{A},{ }_{j}=0$

that is, the symmetric and anti-symmetric parts of an entire stress field in equilibrium, are individually in equilibrium. It is also seen by direct inspection of (12) that the second derivatives of displacement are commutative.

The procedure to prove domain independence is now applied to the decomposition scheme. Substitution of $(11,12)$ in the integrand of $(10)$ and rearranging yield

$$
\begin{gathered}
\sigma_{i j} u_{i},{ }_{j l}-\sigma_{i j},{ }_{j} u_{i}, l-\sigma_{j i} u_{i},{ }_{l j} \\
=\sum_{M, N} \sigma_{i j}^{M} u_{i}^{N},{ }_{j l}-\sigma_{i j}^{M},{ }_{j} u_{i}^{N},{ }_{l}-\sigma_{j i}^{M} u_{i}^{N},_{l j} \\
M, N=S, A
\end{gathered}
$$

in which explicit use has been made of (14).

Equation 15 is a sum of four terms, one symmetric ( $M, N=S, S$, etc.), two mixed symmetric-anti-symmetric and one anti-symmetric term or terms. All four terms vanishes independently at any point because all critical conditions for domain independence are individually fulfilled in every term.

Details of the integration procedure of (15) might differ according to the particular application. The integration procedure is thus most conveniently dealt with in its context and is therefore described below in relation to the specific application. Integrating (15), that is, integrating on the entire field, results in a sum of four domain independent integrals. Contribution from the mixed parts to the four integrals is however cancelled through integration along paths that are symmetric with respect to the $x_{1}$-axis (ibid.), leaving a resulting sum of the symmetric and anti-symmetric parts only. Decomposition of the entire fields before integration allows treatment of the symmetric part, or the Mode I case, and the anti-symmetric part, the Mode II and Mode III cases, individually.

Further decomposition of the anti-symmetric part into Mode II and III takes another route, because, first, in this case there is no counterpart to the equilibrium equations (14) of prospective decomposed parts. 
To decompose the anti-symmetric fields, let $\sigma_{i j}^{\mathrm{II}}$ be Modus II stress and $\sigma_{i j}^{\mathrm{III}}$ be Modus III stress, etc., Then the sum of the Modus II and III parts equals the antisymmetric part.

$\sigma_{i j}^{A}=\sigma_{i j}^{\mathrm{II}}+\sigma_{i j}^{\mathrm{III}}$

$u_{i}^{A}=u_{i}^{\mathrm{II}}+u_{i}^{\mathrm{III}}$

In the same manner as for the entire field, substitution of $(16,17)$ in the integrand of $(10)$ and rearranging yield

$$
\begin{aligned}
\sigma_{i j}^{A} & u_{i}^{A},{ }_{j l}-\sigma_{i j}^{A},{ }_{j} u_{i}^{A},{ }_{l}-\sigma_{j i}^{A} u_{i}^{A},{ }_{l j} \\
= & \sigma_{i j}^{\mathrm{II}} u_{i}^{\mathrm{II}},{ }_{j l}+\sigma_{i j}^{\mathrm{II}} u_{i}^{\mathrm{III}},{ }_{j l}+\sigma_{i j}^{\mathrm{III}} u_{i}^{\mathrm{II}},{ }_{j l}+\sigma_{i j}^{\mathrm{III}} u_{i}^{\mathrm{III},}{ }_{j l} \\
& -\left(\sigma_{i j}^{\mathrm{II}}+\sigma_{i j}^{\mathrm{III}}\right),{ }_{j}\left(u_{i}^{\mathrm{II}}+u_{i}^{\mathrm{III}}\right),{ }_{l} \\
& -\left(\sigma_{j i}^{\mathrm{II}} u_{i}^{\mathrm{II}},{ }_{l j}+\sigma_{j i}^{\mathrm{II}} u_{i}^{\mathrm{III}},{ }_{l j}+\sigma_{j i}^{\mathrm{III}} u_{i}^{\mathrm{II}},{ }_{l j}+\sigma_{j i}^{\mathrm{III}} u_{i}^{\mathrm{III}},{ }_{l j}\right)
\end{aligned}
$$

the difference from (15) being that the equilibrium equation for the entire anti-symmetric stress field, $\sigma_{i j}^{A},{ }_{j}=$ $\left(\sigma_{i j}^{\mathrm{II}}+\sigma_{i j}^{\mathrm{III}}\right),_{j}=0$, cannot be decomposed directly.

The decomposition of the anti-symmetric stress $\sigma^{A}$ into Mode II and Mode III is

$$
\sigma^{A}=\left[\begin{array}{ccc}
\sigma_{11}^{\mathrm{II}} & \sigma_{12}^{\mathrm{II}} & 0 \\
\cdots & \sigma_{22}^{\mathrm{II}} & 0 \\
\text { sym. } & \cdots & \sigma_{33}^{\mathrm{II}}
\end{array}\right]+\left[\begin{array}{ccc}
0 & 0 & \sigma_{13}^{\mathrm{III}} \\
\cdots & 0 & \sigma_{23}^{\mathrm{III}} \\
\text { sym. } & \cdots & 0
\end{array}\right]
$$

In order to obtain harmonizing plane strain conditions for Mode I and Mode II, particularly in mixed mode states (the denominators of $K_{\mathrm{I}}^{2}$ and $K_{\mathrm{II}}^{2}$ in (1) cannot be different for a given mode), the normal stress $\sigma_{33}^{A}$ is taken as a Mode II stress and also because there is no such stress in Mode III, at least not in the crack tip near region (Irwin 1957). The shear stresses $\sigma_{13}^{A}$ and $\sigma_{23}^{A}$ are taken as Mode III stresses for obvious reason. Further, in order to proceed in like manner as for the symmetric-anti-symmetric decomposition, a possible solution is to require $\sigma_{i 3}^{A}$ to be constant in the $x_{3}$-direction, that is, $\sigma_{i 3}^{A},_{3}=0$, which amounts to equilibrium for Modus II stresses, or $\sigma_{i j}^{\mathrm{II}},{ }_{j}=0$ for $i, j=1,2$. Explicitly we get the decomposition of the equilibrium equation of the anti-symmetric stress $\sigma_{i j}^{A},{ }_{j}=0$ :

$$
\begin{aligned}
& \sigma_{11}^{\mathrm{II},{ }_{1}}+\sigma_{12}^{\mathrm{II},{ }_{2}}=0 \quad \sigma_{13}^{\mathrm{III},{ }_{3}}=0 \\
& \sigma_{21}^{\mathrm{II},{ }_{1}}+\sigma_{22}^{\mathrm{II}},_{2}=0 \quad \sigma_{23}^{\mathrm{III},{ }_{3}}=0 \\
& \sigma_{31}^{\mathrm{III},{ }_{1}}+\sigma_{32}^{\mathrm{III}},_{2}=0 \quad \sigma_{33,3}^{\mathrm{II}}=0
\end{aligned}
$$

Now, substitution of the equilibrium equations (20) in the integrand expression (18) results in

$$
\begin{gathered}
\sigma_{i j}^{A} u_{i}^{A},{ }_{j l}-\sigma_{i j}^{A},{ }_{j} u_{i}^{A},_{l}-\sigma_{j i}^{A} u_{i}^{A},{ }_{l j} \\
=\sum_{M, N} \sigma_{i j}^{M} u_{i}^{N},{ }_{j l}-\sigma_{i j}^{M},{ }_{j} u_{i}^{N},_{l}-\sigma_{j i}^{M} u_{i}^{N},{ }_{l j} \\
\quad M, N=\text { II, III }
\end{gathered}
$$

and it remains to satisfy (9).

The stress decomposition (19) implies the displacement derivative decomposition

$$
\begin{aligned}
u_{i}^{A},{ }_{j}= & u_{i}^{\mathrm{II}},{ }_{j}+u_{i}^{\mathrm{III}},_{j} \\
= & \frac{1}{2}\left[\begin{array}{c}
\left(u_{1}-u_{1}^{\prime}\right),{ }_{j} \\
\left(u_{2}+u_{2}^{\prime}\right),_{j} \\
0
\end{array}\right] \\
& +\frac{1}{2}\left[\begin{array}{c}
0 \\
0 \\
\left(u_{3}-u_{3}^{\prime}\right),{ }_{j}
\end{array}\right] j=1,2
\end{aligned}
$$

$$
\begin{aligned}
u_{i}^{A},_{3}=u_{i}^{\mathrm{II}},_{3}+u_{i}^{\mathrm{III}},_{3}= & \frac{1}{2}\left[\begin{array}{c}
0 \\
0 \\
\left(u_{3}-u_{3}^{\prime}\right),_{3}
\end{array}\right] \\
& +\frac{1}{2}\left[\begin{array}{c}
\left(u_{1}-u_{1}^{\prime}\right),{ }_{3} \\
\left(u_{2}+u_{2}^{\prime}\right),{ }_{3} \\
0
\end{array}\right]
\end{aligned}
$$

and (9) imposes the restrictions for Mode II and III

$$
\begin{aligned}
\left(u_{1}-u_{1}^{\prime}\right),{ }_{j 3} & =\left(u_{2}+u_{2}^{\prime}\right),{ }_{j 3} \\
& =\left(u_{3}-u_{3}^{\prime}\right){ }_{3 j}=0
\end{aligned}
$$

or

$$
\begin{aligned}
\left(u_{1}-u_{1}^{\prime}\right)_{3} & =\left(u_{2}+u_{2}^{\prime}\right),_{3}=\left(u_{3}-u_{3}^{\prime}\right),_{3} \\
& =\text { const. }
\end{aligned}
$$

Thus the anti-symmetric displacements $u_{i}^{A}$ may vary linearly in the $x_{3}$-direction which for $u_{3}^{A}$ in particular also can be stated as $\varepsilon_{33}^{A}=u_{3}^{A},_{3}=$ const. These conditions do not imply any further restrictions beyond those associated with (20).

Integrating (21), that is, integrating on the antisymmetric field, would result in sum of four domain independent integrals. Contribution from the mixed parts is again avoided by integration along paths that are symmetric with respect to the $x_{1}$-axis, leaving a sum of the Mode II and Mode III parts. As before, decomposition of the anti-symmetric field before integration 
allows calculation of the Mode II and Mode III parts individually.

We are now in position to define the Mode III Eshelby tensor as

$P_{l j}^{\mathrm{III}}=W^{\mathrm{III}} \delta_{l j}-\sigma_{j i}^{\mathrm{III}} u_{i}^{\mathrm{III}},_{l}$

in which

$$
\begin{aligned}
W^{\mathrm{III}}= & \int_{0}^{\varepsilon_{i j}} \sigma_{i j}^{\mathrm{III}} \mathrm{d} \varepsilon_{i j}^{\mathrm{III}}=2 \int_{0}^{\varepsilon_{13}^{\mathrm{III}}} \sigma_{13}^{\mathrm{III}} \mathrm{d} \varepsilon_{13}^{\mathrm{III}} \\
& +2 \int_{0}^{\varepsilon_{23}^{\mathrm{III}}} \sigma_{23}^{\mathrm{III}} \mathrm{d} \varepsilon_{23}^{\mathrm{III}}
\end{aligned}
$$

on account of symmetry of stress and strain, and where

$\varepsilon_{i j}^{\mathrm{III}}=1 / 2\left(u_{i}^{\mathrm{III}},{ }_{j}+u_{j}^{\mathrm{III}},_{i}\right)$

in general and in particular

$\varepsilon_{13}^{\mathrm{III}}=1 / 2\left(\left(u_{1}-u_{1}^{\prime}\right),_{3}+\left(u_{3}-u_{3}^{\prime}\right),_{1}\right)$

$\varepsilon_{23}^{\mathrm{III}}=1 / 2\left(\left(u_{2}+u_{2}^{\prime}\right),_{3}+\left(u_{3}-u_{3}^{\prime}\right),,_{2}\right)$.

An application of (24) to a stress state which is not constant in the $x_{3}$-direction will thus suffer from errors related to the $x_{3}$-stress gradients. It is reasonable to expect that these are much smaller than the in-plane stress gradients in the crack tip near region. This means that the Mode II and III equilibrium equations are approximately satisfied and in turn that the error is likely to be small near the crack tip but will increase for an extended integration domain.

This decomposition scheme is in agreement with that in Rigby and Aliabadi (1998) but regarding the stress $\sigma_{33}^{A}$ in disagreement with others (e.g. Nikishkov and Atluri 1987; Shivakumar and Raju 1992).

Decomposition obtained, for Mode III we now proceed to two new applications.

\section{Path independent integrals for the crack extension force of a plane circular crack in axi-symmetric in-plane and Mode III loading}

Consider a 3D-body with a plane circular crack and traction-free crack surfaces. Let $(r, \varphi, z)$ be cylinder coordinates with $z$ normal to the crack plane and origin at the centre of the crack.
Eshelby's energy-momentum tensor reads in curvilinear coordinates

$P_{l}^{j}=W \delta_{l}^{j}-\left.\sigma^{i j} u_{i}\right|_{l}$

where ( $)\left.\right|_{l}$ denotes a covariant derivative. The notation in sub- and superscripts is not essential as cylinder coordinates are orthogonal, but is retained foremost because reduction of tensors to their corresponding physical quantities is facilitated (See also Appendix for reduction to physical quantities).

The path independent integral expression in this section are determined with a virtual crack extension method, which is fully described in Eriksson (2002), and in which $\delta \gamma^{i}$ is a virtual displacement in the body which describes crack extension, for the time being along a prospective crack front. In the absence of a crack, $\left.P_{i}^{j}\right|_{j}=0$ by (4) and from this it follows that also the summed factors $\left.\delta \gamma^{i} P_{i}^{j}\right|_{j}$ vanish independently of the choice of $\delta \gamma^{i}$. To simplify derivation of the desired result below, the sum $\left.\delta \gamma^{i} P_{i}^{j}\right|_{j}$ is premultiplied by $1 / r$. Then, for a volume $V$ with surface $S$, the integral

$\left.\int_{V} \frac{1}{r} \delta \gamma^{i} P_{i}^{j}\right|_{j} \mathrm{~d} V$

vanishes independently of $V$ and the integral and its transformations are path or domain independent for $r>$ 0 . Integrating (28) by parts yields

$$
\begin{aligned}
& \left.\int_{V}\left(\frac{1}{r} \delta \gamma^{i} P_{i}^{j}\right)\right|_{j} \mathrm{~d} V-\left.\int_{V} \frac{1}{r} P_{i}^{j} \delta \gamma^{i}\right|_{j} \mathrm{~d} V \\
& -\left.\int_{V} P_{i}^{j} \delta \gamma^{i} \frac{1}{r}\right|_{j} \mathrm{~d} V
\end{aligned}
$$

The integrands are scalars and by applying Gauss' divergence theorem to the first integral, (29) is transformed into

$$
\begin{aligned}
& \int_{S} \frac{1}{r} \delta \gamma^{i} P_{i}^{j} n_{j} \mathrm{~d} S-\left.\int_{V} \frac{1}{r} P_{i}^{j} \delta \gamma^{i}\right|_{j} \mathrm{~d} V \\
& -\left.\int_{V} P_{i}^{j} \delta \gamma^{i} \frac{1}{r}\right|_{j} \mathrm{~d} V
\end{aligned}
$$

where $n_{j}$ is the outward positive unit normal vector of $S$.

In cylinder coordinates the only non-zero Christoffel symbols are (e.g. Fung and Tong 2001)

$\Gamma_{\varphi \varphi}^{r}=-r \quad$ and $\quad \Gamma_{r \varphi}^{\varphi}=\Gamma_{\varphi r}^{\varphi}=1 / r$ 
Let the virtual displacement be $\left(\delta \gamma^{r}, 0,0\right)$ and keep the physical quantity $\delta \hat{\gamma}_{r}=\delta \gamma^{r}$ a constant to model prospective crack extension in the $r$-direction. Then the covariant derivative in the second integral of (30) is (see Appendix A3)

$$
\left.\delta \gamma^{i}\right|_{j}=\delta \gamma^{r} \Gamma_{r j}^{i}
$$

and the only non-zero component of this derivative is

$$
\left.\delta \gamma^{\varphi}\right|_{\varphi}=\delta \gamma^{k} \Gamma_{r \varphi}^{\varphi}=\delta \gamma^{r} / r
$$

In the third integral in (30) we have

$$
\left.\frac{1}{r}\right|_{j}=\frac{\partial}{\partial \theta_{j}}\left(\frac{1}{r}\right)=-\frac{1}{r^{2}} \text { for } j=r
$$

Substitution of $(33,34)$ in $(30)$ yield

$$
\begin{aligned}
0= & \int_{S} \frac{1}{r} P_{r}^{j} \delta \gamma^{r} n_{j} \mathrm{~d} S-\int_{V} \frac{1}{r^{2}} P_{\varphi}^{\varphi} \delta \gamma^{r} \mathrm{~d} V \\
& -\int_{V} \frac{1}{r^{2}} P_{r}^{r} \delta \gamma^{r} \mathrm{~d} V \quad j=r, \varphi, z
\end{aligned}
$$

for prospective radial crack extension. Now introduce a crack with radius $r=a$ and let the volume $V$, with surface $S$, be a tubular slice in a small sector $\Delta \varphi$, with a radial cut and located around a part of the crack such that the inside of the tube surrounds but does not include any part of the crack front and the crack plane enters the cut. The surface $S$ is separated into six parts, Fig. 1, so that

$S=S_{i}+S_{c}^{ \pm}+S_{e}^{ \pm}+S_{o}$

Here $S_{i}$ is a torus-shaped inner surface around and arbitrarily close to the crack front and $S_{o}$ an outer surface of the same shape but at a finite distance from the crack front, none dependent on $\varphi$. Let $\Gamma$ and $\Gamma_{i}$

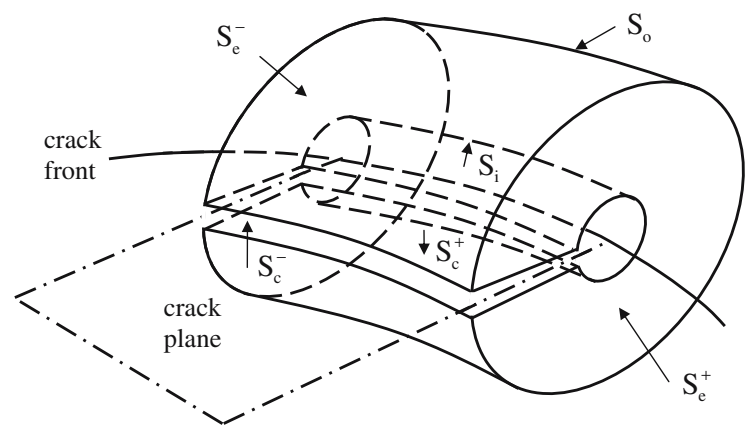

Fig. 1 Surface partition of the sliced tubular region with volume $V$ and surface $S$ be curves formed by the section of $S_{o}$ and $S_{i}$, respectively, with the plane $\varphi=$ const. The curves $\Gamma$ and $\Gamma_{i}$ are taken positive clock-counter-wise in their plane. On $S_{i}$ and $S_{o}$ we then have $\mathrm{d} S=r \Delta \varphi \mathrm{d} \Gamma$ where $r \Delta \varphi$ is an element of length along the $\varphi$-coordinate and $\mathrm{d} \Gamma$ an element of length along $\Gamma$ or $\Gamma_{i}$. Further we have that $d V=r \Delta \varphi d A$ where $d A$ is a surface element in the plane $\varphi=$ const. As the closed surface $S$ does not contain the crack front (and related singularities) the region inside $S$ is regular and the transformation (30) holds there.

The surfaces $S_{e}^{ \pm}$are the end-surfaces of the tubular volume $V$, with normal vector $\left(0, \pm n_{\varphi}, 0\right)$. This means that only $P_{r}^{\varphi}$ contributes to the surface integrals on $S_{e}^{ \pm}$, but for the load cases considered in this work, in axi-symmetric loading

$$
\begin{aligned}
P_{r}^{\varphi} & =0-\left.\sigma^{\varphi i} u_{i}\right|_{r} \\
& =-\left(\left.\sigma^{\varphi r} u_{r}\right|_{r}+\left.\sigma^{\varphi \varphi} u_{\varphi}\right|_{r}+\left.\sigma^{\varphi z} u_{z}\right|_{r}\right) \\
& =-\left(\left.0 u_{r}\right|_{r}+\sigma^{\varphi \varphi} 0+\left.0 u_{z}\right|_{r}\right)=0
\end{aligned}
$$

and in Mode III loading

$P_{r}^{\varphi}=-\left(\sigma^{\varphi r} 0+\left.0 u_{\varphi}\right|_{r}+\sigma^{\varphi z} 0\right)=0$

(see (24) and Appendix A5).

Thus, the surface integrals on $S_{e}^{ \pm}$vanish in both cases. The singular properties of the stress and strain fields at the crack tip do not enter the calculation through the surface integrals.

$S_{c}^{ \pm}$are the two crack surfaces with normal vector $\left(0,0, \pm n_{z}\right)$, respectively. Here, only $P_{r}^{z}$ contributes, but with Cauchy's formula $t^{i}=\sigma^{i j} n_{j}$ substituted

$$
\begin{aligned}
& P_{r}^{z} n_{z}=\left(0-\left.\sigma^{z i} u_{i}\right|_{r}\right) n_{z} \\
& =-\left(\left.\sigma^{z r} u_{r}\right|_{r}+\left.\sigma^{z \varphi} u_{\varphi}\right|_{r}+\left.\sigma^{z z} u_{z}\right|_{r}\right) n_{z}
\end{aligned}
$$

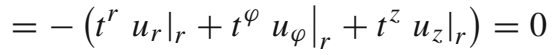

as the stress vector $t^{i}=0$ on a stress-free surface. Stress-free surfaces apply to both axi-symmetric and Mode III loading.

Substitution of $(37,38)$ in $(35)$ results in

$$
\begin{gathered}
\int_{\Gamma_{i}} P_{r}^{j} n_{j} \mathrm{~d} \Gamma=\int_{\Gamma} P_{r}^{j} n_{j} \mathrm{~d} \Gamma-\int_{A} \frac{1}{r}\left(P_{\varphi}^{\varphi}-P_{r}^{r}\right) \mathrm{d} A \\
j=r, z
\end{gathered}
$$

after cancelling of $\delta \gamma^{r} \Delta \varphi$. Here $r \Delta \varphi$ is taken small but finite and so small that all quantities inside $V$ can be considered constant in the $\varphi$-direction. Equation 39 can be seen as a balance equation that holds for an arbitrary, or in particular, a unit radial crack extension. 
With $\Gamma_{i}$ arbitrarily close to the crack tip we have in the limit

$$
J=\lim _{\Gamma_{i} \rightarrow 0} \int_{\Gamma_{i}} P_{r}^{j} n_{j} \mathrm{~d} \Gamma \quad j=r, z
$$

(e.g. Moran and Shih 1987) per unit length of the crack front and where

$$
\int_{\Gamma_{i}} P_{r}^{j} n_{j} \mathrm{~d} \Gamma=\int_{\Gamma_{i}}\left(W n_{r}-t^{i} u_{i, r}\right) \mathrm{d} \Gamma \quad j=r, z
$$

The $P_{l}^{j}$-components in the area integral are

$$
\begin{aligned}
P_{\varphi}^{\varphi} & =W-\left.\sigma^{\varphi i} u_{i}\right|_{\varphi} \\
& =W-\left(\left.\sigma^{\varphi r} u_{r}\right|_{\varphi}+\left.\sigma^{\varphi \varphi} u_{\varphi}\right|_{\varphi}+\left.\sigma^{\varphi z} u_{z}\right|_{\varphi}\right) \\
P_{r}^{r} & =W-\left.\sigma^{r i} u_{i}\right|_{r} \\
& =W-\left(\left.\sigma^{r r} u_{r}\right|_{r}+\left.\sigma^{r \varphi} u_{\varphi}\right|_{r}+\left.\sigma^{r z} u_{z}\right|_{r}\right)
\end{aligned}
$$

For an axi-symmetrically in-plane loaded plane circular crack the $P_{l}^{j}$-components are

$$
\begin{aligned}
& P_{\varphi}^{\varphi}=W-\left.\sigma^{\varphi \varphi} u_{\varphi}\right|_{\varphi} \\
& P_{r}^{r}=W-\left.\sigma^{r r} u_{r}\right|_{r}-\left.\sigma^{r z} u_{z}\right|_{r}
\end{aligned}
$$

(see Appendix). From (39) to (42) is obtained

$$
\begin{aligned}
J= & \int_{\Gamma}\left(W n_{r}-\left.t^{i} u_{i}\right|_{r}\right) \mathrm{d} \Gamma \\
& -\int_{A} \frac{1}{r}\left(\left.\sigma^{r r} u_{r}\right|_{r}-\left.\sigma^{\varphi \varphi} u_{\varphi}\right|_{\varphi}+\left.\sigma^{r z} u_{z}\right|_{r}\right) \mathrm{d} A \\
& \quad j=r, z
\end{aligned}
$$

which after transformation into physical quantities results in

$$
\begin{aligned}
J= & \int_{\Gamma}\left(W \hat{n}_{r}-\hat{t}_{i} \hat{u}_{i ; r}\right) \mathrm{d} \Gamma \\
& -\int_{A} \frac{1}{r}\left(\hat{\sigma}_{r r} \hat{u}_{r, r}-\hat{\sigma}_{\varphi \varphi} \hat{u}_{\varphi, \varphi}+\hat{\sigma}_{r z} \hat{u}_{z, r}\right) \mathrm{d} A \\
& \quad j=r, z
\end{aligned}
$$

This expression has previously been obtained by several (Bergkvist and Lan Huong 1977; Broberg 1999), each through different routes, and is included here to show applicability of the present method and agreement with known results.
3.1 Mode III loading

For an axi-symmetrically loaded plane circular Mode III crack the $P_{l}^{j}$-components are

$P_{\varphi}^{\varphi}=W-\left.\sigma^{\varphi r} u_{r}\right|_{\varphi}$

$P_{r}^{r}=W-\left.\sigma^{r \varphi} u_{\varphi}\right|_{r}$

which yield

$P_{\varphi}^{\varphi}-P_{r}^{r}=\sigma^{\varphi r}\left(\left.u_{\varphi}\right|_{r}-\left.u_{r}\right|_{\varphi}\right)=\sigma^{\varphi r} \frac{\partial u_{\varphi}}{d r}$

(see Appendix A12) and through rearrangement of (43) is obtained

$$
\begin{aligned}
J= & \int_{\Gamma}\left(W n_{r}-\left.\sigma^{j i} u_{i}\right|_{r} n_{j}\right) \mathrm{d} \Gamma \\
& -\int_{A} \frac{1}{r} \sigma^{r \varphi} \frac{\partial u_{\varphi}}{\partial r} \mathrm{~d} A \quad j=r, z
\end{aligned}
$$

which after transformation into physical quantities results in

$$
\begin{aligned}
J= & \int_{\Gamma}\left(W \hat{n}_{r}-\hat{t}_{i} \hat{u}_{i ; r}\right) \mathrm{d} \Gamma \\
& -\int_{A} \frac{1}{r} \hat{\sigma}_{r \varphi}\left(\frac{\partial \hat{u}_{\varphi}}{\partial r}+\frac{\hat{u}_{\varphi}}{r}\right) \mathrm{d} A \quad j=1,3
\end{aligned}
$$

(see Appendix A15). The last integral in (48) can be seen as a correction term due to curvature. This term decreases with increasing radius of curvature and tends in the limit to zero as the crack front approaches a straight line and the original formulation of the $J$-integral (Rice 1968) is recovered.

\section{Domain integral formulation}

A derivation of the domain integral formulation of the crack extension force is first briefly sketched. Let $q^{i}$ be a prescribed virtual displacement field, satisfying certain general and boundary conditions, and to be explicitly used in computation. From (28) it follows that in the absence of a crack, the integral

$\left.\int_{V} q^{i} P_{i}^{j}\right|_{j} \mathrm{~d} V$

is domain independent. Integration by parts yield

$$
\left.\int_{V}\left(q^{i} P_{i}^{j}\right)\right|_{j} \mathrm{~d} V-\left.\int_{V} P_{i}^{j} q^{i}\right|_{j} \mathrm{~d} V
$$


As the integrands are scalars, we may use curvilinear coordinates and the divergence theorem. The latter applied to the first integral in (50) yields

$$
\int_{S} q^{i} P_{i}^{j} n_{j} \mathrm{~d} S-\left.\int_{V} P_{i}^{j} q^{i}\right|_{j} \mathrm{~d} V
$$

The volume $V$ is now given a tubular shape and located around a part of a crack as described in Sect. 3. The surface $S$ is in like manner separated into six different parts. Several assumptions regarding the form of the virtual displacement field exist (e.g. deLorenzi 1982; Shih et al. 1986) (where also more rigorous derivations of the domain integral formulation can be found). A virtual displacement field with just one component in the direction of crack extension, but itself a function of all three coordinates, is found to be sufficient in general (Shivakumar and Raju 1992), or

$$
\left(q^{r}(r, \varphi, z), 0,0\right)
$$

for radial crack extension. At the crack tip the mean value of the physical component of $q^{r}$ along the crack front is unity. On the surfaces $S_{o}$ and $S_{e}^{ \pm}$the displacement $q^{r}$ is set to zero. In between $q^{r}$ may be any smooth continuous function of position. In practice $q^{r}$ is at most parabolic along the crack front and linear in radial directions (ibid.).

As $q^{r}$ is perpendicular to the normal vector on the crack surfaces, which also are assumed stress-free, the surface integrals on the crack surfaces are zero and do not contribute to (51). The remaining integral on $S_{i}$, with $d S=l d \Gamma$, where $l$ is the length of $S_{i}$ along the crack front, can be written

$$
-l \int_{\Gamma_{i}} \hat{P}_{r}^{j} \hat{n}_{j} \mathrm{~d} \Gamma \quad j=r, z
$$

Thus, in the limit as $\Gamma_{i}$ tends to zero in (53), we get from (40a, 52), with $V$ not changed

$$
J l=-\left.\int_{V} P_{i}^{j} q^{i}\right|_{j} \mathrm{~d} V
$$

where $J$ is a mean value of the crack extension force over $l$.

Substitution of $(27,52)$ into the integrand of $(54)$, expanding and collecting terms with respect to Christoffel symbols yield

$W \frac{\partial q^{r}}{\partial r}-\left.\sigma^{j k} u_{k}\right|_{i} \frac{\partial q^{i}}{\partial \theta^{j}}+q^{r}\left(W \Gamma_{r i}^{i}-\left.\sigma^{j k} u_{k}\right|_{i} \Gamma_{r j}^{i}\right)$

The first two terms in (55) represent the domain integral integrand in Cartesian coordinates and yield the contribution to $J$ for a straight crack front. The last term can be seen as a correction term due to crack curvature. This term, expanded and collected with respect to the Christoffel symbols, is

$$
\begin{aligned}
q^{r} & \left(\left(W-\left.\sigma^{r k} u_{k}\right|_{r}\right) \Gamma_{r r}^{r}+\left(W-\left.\sigma^{\varphi k} u_{k}\right|_{\varphi}\right) \Gamma_{r \varphi}^{\varphi}\right. \\
& \left.-\left(\left.\sigma^{r k} u_{k}\right|_{\varphi} \Gamma_{r r}^{\varphi}+\left.\sigma^{\varphi k} u_{k}\right|_{r} \Gamma_{r \varphi}^{r}\right)\right)
\end{aligned}
$$

or shorter

$q^{r}\left(P_{r}^{r} \Gamma_{r r}^{r}+P_{\varphi}^{\varphi} \Gamma_{r \varphi}^{\varphi}-\left(P_{\varphi}^{r} \Gamma_{r r}^{\varphi}+P_{r}^{\varphi} \Gamma_{r \varphi}^{r}\right)\right)$

In cylinder coordinates the only non-zero Christoffel symbols are $\Gamma_{\varphi \varphi}^{r}$ and $\Gamma_{r \varphi}^{\varphi}=\Gamma_{\varphi r}^{\varphi}$ which for orthogonal coordinates can be written (Eriksson 2000)

$\Gamma_{\varphi \varphi}^{r}=-\frac{g_{r r}, \varphi}{2 g_{\varphi \varphi}}, \quad \Gamma_{r \varphi}^{\varphi}=\Gamma_{\varphi r}^{\varphi}=\frac{g_{\varphi \varphi, r}}{2 g_{\varphi \varphi}}$

where ( ) ${ }_{i}$ denotes the partial derivative $\partial() / \partial \theta^{k}$. The tensor component $q^{r}$ and its physical quantity $\hat{q}_{r}$ are related through

$q^{r}=\hat{q}_{r} / \sqrt{g_{r r}}$

Substitution of $(57,58)$ into $(56)$ gives

$\hat{q}_{r} P_{\varphi}^{\varphi} \frac{g_{\varphi \varphi, r}}{2 g_{\varphi \varphi} \sqrt{g_{r r}}}$

The radius of curvature in the plane $z=$ const. of a curve $r=$ const. is (ibid.)

$\frac{1}{R_{\varphi r}}=\frac{g_{\varphi \varphi, r}}{2 g_{\varphi \varphi} \sqrt{g_{r r}}}$

The effect of crack curvature thus enters only through (the inverse of) the radius of curvature. The adjustment due to curvature decreases with radius of curvature and approaches zero as the radius of curvature tends to infinity, which is the radius of curvature of a straight crack.

In cylinder coordinates, $g_{r r}=1$ and $g_{\varphi \varphi}=r^{2}$ (e.g. Fung and Tong 2001), and (59) reduces to

$\hat{q}_{r} P_{\varphi}^{\varphi} \frac{1}{R_{\varphi r}}=\frac{\hat{q}_{r}}{r}\left(W-\left.\sigma^{\varphi k} u_{k}\right|_{\varphi}\right)$

For axi-symmetric loading, with $\sigma^{\varphi r}=\sigma^{\varphi z}=0$, we arrive at the correction term

$\frac{\hat{q}_{r}}{r}\left(W-\sigma^{\varphi \varphi} u_{\varphi, \varphi}\right)=\frac{\hat{q}_{r}}{r}\left(W-\hat{\sigma}_{\varphi \varphi} \hat{u}_{\varphi} ; \varphi\right)$

Here, a semicolon, (;), denotes the physical component of a covariant derivative. Using the notation $\hat{\varepsilon}_{\varphi \varphi}=$ $\hat{u}_{\varphi} ; \varphi$ the domain integral form of $J$ can be written

$J l=\int_{V}\left(\hat{\sigma}_{j k} \hat{u}_{k} ; r \frac{\partial \hat{q}_{r}}{\partial \theta^{j}}-W \frac{\partial \hat{q}_{r}}{\partial r}-\frac{\hat{q}_{r}}{r}\left(W-\hat{\sigma}_{\varphi \varphi} \hat{\varepsilon}_{\varphi \varphi}\right)\right) \mathrm{d} V$ 
This result, which previously has been obtained by deLorentzi (1985) and Shih et al. (1986) is included, again to illustrate the applicability of the method and agreement with previous results.

\subsection{Mode III loading}

Turning attention to Mode III loading, the corresponding integral for Mode III can now be obtained straightforwardly. In Mode III we have $\sigma^{\varphi \varphi}=0$ and $u_{z} ; \varphi=0$. Modifying (62) accordingly results in

$\frac{\hat{q}_{r}}{r}\left(W-\left.\sigma^{\varphi r} u_{r}\right|_{\varphi}\right)=\frac{\hat{q}_{r}}{r}\left(W-\hat{\sigma}_{\varphi r} \hat{u}_{\varphi} / r\right)$

for the curvature correction term. The domain integral form of $J$ in Mode III is finally obtained through rearrangement of (63) as

$$
\begin{aligned}
J l= & \int_{V}\left(\hat{\sigma}_{j \varphi} \hat{u}_{\varphi} ; r \frac{\partial \hat{q}_{r}}{\partial \theta^{j}}-W \frac{\partial \hat{q}_{r}}{\partial r}-\frac{\hat{q}_{r}}{r}\left(W-\hat{\sigma}_{\varphi r} \frac{\hat{u}_{\varphi}}{r}\right)\right) \\
& \mathrm{d} V \quad j=r, z
\end{aligned}
$$

Also in this case the curvature correction term tends to zero as the radius of curvature tends to infinity.

\section{Discussion}

The first of the new Mode III solutions, (48), consists of a path and an area integral and the second, (65), is a volume or domain integral. Both types of solutions comprise a part that applies to a crack with a straight front, viz. the path integral only in (48) and an expression that includes only the first two terms of the integrand in (65). The remainder in each solution, the area integral in (48), and the last two terms (including their factor) of the integrand in (65), can be seen as correction terms due to crack curvature.

For the first solution, (48), to exist, it must have a finite limit value when integrated on a small circle around the crack tip whose radius $\rho$ tends to zero (where $\rho$ is radial distance from the crack tip in a plane $\varphi=$ constant). This condition is fulfilled if the path integral tends to the limit value and the area integral tends to zero. (Note here that $r$ in this limiting process tends to a.) From this we conclude that the quantity inside the bracket in the path integral must be of $\operatorname{order} \rho^{-1}$. As the integrand of the area integral is of the same order or weaker, this integral yields the desired limit value.
Such singular behaviour at a crack tip is found e.g. for linear and non-linear elastic materials and power-law hardening materials (Andersson 1995). Further, the singularity in the integrand of the area integral in (48) also appears in previous solutions (44). This means that (48) exists under the same condition as for (44) and similar solutions, e.g. (Amestoy et al. 1981). Thus for materials fulfilling the crack tip singularity condition the integral (48) tends to a limit for any admissible integration path.

A similar argument goes for the second solution. For this solution, (65), to exist, it must have the finite limit value (40a) per unit length along the crack front, when integrated on a vanishingly small circle around the crack tip. It is not difficult to show that this condition is fulfilled (for any admissible virtual displacement field) if $P_{i}^{j}$ is of the order $\rho^{-1}$, as in this case the part of (65) corresponding to the curvature correction term tends to zero. Further, the singularity in the correction term also appears in the corresponding term of deLorentzi's (1985) original solution and (65) thus exists under the same integrability conditions as (63). The pertinent conditions of integrability are given in more detail in (ibid).

\section{Summary}

In this work, a decomposition scheme of Eshelby's energy momentum tensor is presented, which results in zero divergence decomposed parts and allows formulation of expressions for Mode I, II and III crack tip parameters, with particular emphasis on Mode III. By using the Mode III decomposition and the virtual crack extension method, a path and a domain independent integral for the crack extension force of a plane circular crack in axisymmetric Mode III loading are derived.

\section{Appendix}

\section{Covariant derivatives}

For convenience, the only non-zero Christoffel symbols in cylinder coordinates (Fung and Tong 2001) are repeated here:

$\Gamma_{\varphi \varphi}^{r}=-r \quad$ and $\quad \Gamma_{r \varphi}^{\varphi}=\Gamma_{\varphi r}^{\varphi}=1 / r$ 
The covariant derivative of a contravariant vector is

$$
\left.\delta \gamma^{l}\right|_{j}=\frac{\partial \delta \gamma^{l}}{\partial \theta^{j}}+\delta \gamma^{k} \Gamma_{k j}^{l}
$$

The virtual displacement $\delta \hat{\gamma}_{r}=\delta \gamma^{r}=$ const. yields

$$
\left.\delta \gamma^{l}\right|_{j}=\delta \gamma^{k} \Gamma_{k j}^{l}=\delta \gamma^{r} \Gamma_{r j}^{l}
$$

The covariant derivative of a covariant vector is

$$
\left.\delta \gamma_{l}\right|_{j}=\frac{\partial \delta \gamma_{l}}{\partial \theta^{j}}-\delta \gamma_{k} \Gamma_{l j}^{k}
$$

Thus in cylinder coordinates $\left(\theta_{1}, \theta_{2}, \theta_{3}\right)=(r, \varphi, z)$

$$
\begin{aligned}
\left.u_{\varphi}\right|_{r}= & \frac{\partial u_{\varphi}}{\partial r}-u_{k} \Gamma_{\varphi r}^{k}=0 \\
& -\left(u_{r} \Gamma_{\varphi r}^{r}+u_{z} \Gamma_{\varphi r}^{z}\right)=0
\end{aligned}
$$

Stresses, displacements and displacement gradients

In in-plane loading, stress, displacement and displacement gradient are (note renumbering of axes)

$$
\begin{gathered}
\sigma_{i j}(r, z)=\left[\begin{array}{ccc}
\sigma_{r r} & 0 & \sigma_{r z} \\
0 & \sigma_{\varphi \varphi} & 0 \\
\sigma_{z r} & 0 & \sigma_{z z}
\end{array}\right] \\
u_{i}(r, z)=\left(u_{r}, 0, u_{z}\right) \\
\left.u_{i}\right|_{j}=\left[\begin{array}{ccc}
\left.u_{r}\right|_{r} & 0 & \left.u_{r}\right|_{z} \\
0 & \left.u_{\varphi}\right|_{\varphi} & 0 \\
\left.u_{z}\right|_{r} & 0 & \left.u_{z}\right|_{z}
\end{array}\right]
\end{gathered}
$$

and in Mode III loading from (19)

$$
\begin{aligned}
& \sigma_{i j}(r, z)=\left[\begin{array}{ccc}
0 & \sigma_{r \varphi} & 0 \\
\sigma_{\varphi r} & 0 & \sigma_{\varphi z} \\
0 & \sigma_{z \varphi} & 0
\end{array}\right] \\
& u_{i}(r, z)=\left(0, u_{\varphi}, 0\right) \\
& \left.u_{i}\right|_{j}=\left[\begin{array}{ccc}
0 & \left.u_{r}\right|_{\varphi} & 0 \\
\left.u_{\varphi}\right|_{r} & 0 & \left.u_{\varphi}\right|_{z} \\
0 & 0 & 0
\end{array}\right]
\end{aligned}
$$

Further,

$$
\begin{aligned}
\left.u_{\varphi}\right|_{r}-\left.u_{r}\right|_{\varphi} & =\frac{\partial u_{\varphi}}{\partial r}-u_{\varphi} \Gamma_{\varphi r}^{\varphi} \\
-\left(0-u_{\varphi} \Gamma_{r \varphi}^{\varphi}\right) & =\frac{\partial u_{\varphi}}{\partial r}
\end{aligned}
$$

Physical components

The metric tensor in cylinder coordinates is

$$
\left(g_{i j}\right)=\left[\begin{array}{lll}
1 & & \\
& r^{2} & \\
& & 1
\end{array}\right]
$$

and the associated metric tensor

$g^{i j}=1 / g_{i j}$

Then $\hat{u}_{i}=u_{i} \sqrt{g^{\underline{i}} \underline{\underline{i}}}=u^{i} \sqrt{g_{\underline{\underline{i}} \underline{\underline{i}}}}$, where the caret symbol $\left(^{\wedge}\right)$ denotes a physical quantity and an underscored index means no summation. From (46) and the above we get

$$
\begin{aligned}
\sigma^{r \varphi} \frac{\partial u_{\varphi}}{\partial r} & =\frac{\hat{\sigma}_{r \varphi}}{\sqrt{g_{r r}} \sqrt{g_{\varphi \varphi}}} \frac{\partial}{\partial r}\left(\frac{\hat{u}_{\varphi}}{\sqrt{g^{\varphi \varphi}}}\right) \\
& =\hat{\sigma}_{r \varphi} \frac{1}{r}\left(r \frac{\partial \hat{u}_{\varphi}}{\partial r}+\hat{u}_{\varphi}\right)
\end{aligned}
$$

\section{References}

Amestoy M, Bui HD, Labbens R (1981) On the definition of local path independent integrals in three-dimensional crack problems. Mech Res Commun 8:231-236

Andersson TL (1995) Fracture mechanics. Fundamentals and applications. CRC Press, Boca Raton, FL

Bergkvist H, Lan Huong G-L (1977) $J$-integral related quantities in axisymmetric cases. Int J Fract 13:379-386

Broberg KB (1999) Cracks and fracture. Academic Press, London, pp 60-63

deLorentzi HG (1982) On the energy release rate and the $J$ integral for 3-D crack configurations. Int J Fract 19:183193

deLorenzi HG (1985) Energy release rate calculations by the finite element method. Eng Fract Mech 21:129-143

Eriksson K (2000) A general expression for an area integral of a point-wise $J$ for a curved crack front. Int $J$ Fract 106:65-80

Eriksson K (2002) A domain independent integral expression for the crack extension force of a curved crack in three dimensions. J Mech Phys Sol 50:381-403

Eshelby JD (1951) The force on an elastic singularity. Philos Trans R Soc A Lond 244:87-112

Eshelby JD (1970) Energy relations and the energy-momentum tensor in continuum mechanics. In: Kanninen MF (ed) et al Inelastic behaviour of solids. McGraw-Hill, New York, pp 77-115

Fung YC, Tong P (2001) Classical and computational solid mechanics. World Scientific, Singapore

Huber O, Nickel J, Kuhn G (1993) On the decomposition of the J-integral for 3D crack problems. Int J Fract 64:339-348

Irwin GR (1957) Analysis of stress and strains near the end of a crack transversing a plate. J Appl Mech 24:361-364 
Ishikawa H, Kitagawa H, Okamura H (1979) $J$-integral of a mixed mode crack and its application. In: Miller KJ, Smith RF (eds) Proceedings of the 3rd international conference on mechanical behavior of materials. Pergamon Press, Oxford, pp 447-455

Li FZ, Shih CF, Needleman A (1985) A comparison of methods for calculating energy release rates. Eng Fract Mech 21:405-421

Moran B, Shih CF (1987) A general treatment of crack tip contour integrals. Int J Fract 35:295-310

Nikishkov GP, Atluri SN (1987) Calculation of fracture mechanics parameters for an arbitrary three-dimensional crack by the 'Equivalent domain integral' method. Int J Numer Methods Eng 24:1801-1821
Rice JR (1968) A path independent integral and the approximate analysis of strain concentration by notches and cracks. J Appl Mech 35:379-386

Rigby RH, Aliabadi MH (1993) Mixed-mode $J$-integral method for analysis of 3D fracture problems using BEM. Eng Anal Bound Elem 11:239-256

Rigby RH, Aliabadi MH (1998) Decomposition of the mixedmode $J$-integral, revisited. Int J Sol Struct 35:2073-2099

Shih CF, Moran B, Nakamura T (1986) Energy release rate along a three-dimensional crack front in a thermally stressed body. Int J Fract 30:79-102

Shivakumar KN, Raju IS (1992) An equivalent domain integral method for three-dimensional mixed-mode fracture problems. Eng Fract Mech 42:935-959 